\title{
INTRAVESICAL BOTULINUM TOXIN A INJECTIONS IN THE TREATMENT OF PAINFUL BLADDER SYNDROME / INTERSTITIAL CYSTITIS: A SYSTEMATIC REVIEW
}

Hypothesis / aims of study

The aim of our systematic review was to assess the effectiveness and adverse effects of intravesical (BTX-A) in painful bladder syndrome (PBS) or interstitial cystitis (IC).

\section{Study design, materials and methods}

Randomised controlled trials (RCTs) and prospective studies of relevance were identified, assessed for inclusion, then analyzed by two independent reviewers. Newcastle Ottawa scale and Jadad scale were used to assess the qualities of included prospective nonrandomized and RCTs respectively.

\section{Results}

Ten ( 3 RCTs and 7 prospective cohort) studies with a total of 260 participants were included. The studies included patients between the ages of 18 to 83 years [mean age, 49.9 years). The included studies were uniformly small with sample sizes ranging from 10 to 67. Five studies used NIDDK criteria for diagnosing IC, one study used clinical symptoms and presence of sterile urine to diagnose IC, whereas another study used clinical symptoms and cystoscopy to diagnose IC. Preparation of BTXA used was Botox (Allergan, Inc., Irvine, CA, USA) in 5 studies, Dysport (Ipsen Itd., Slough, Berkshire, UK) in 2 studies and was not mentioned in 2 studies. In the two centre case series, Dysport was used to treat Polish patients and Botox was used for US patients. The amount of BTX-A injected was mostly 100-300 U (mostly at a concentration of $10 \mathrm{U} / 1 \mathrm{ml}$ normal saline) except two studies which used $500 \mathrm{U}$ Dysport. In most studies the number of injection sites in the bladder was between 20-30 except in two studies; in one 10 injections performed in the bladder trigone only and in the other study injections in 40 sites in the posterior and lateral walls of the bladder were given. Injection sites included trigone in 4 studies, spared trigone in 2 studies and in the remaining studies it was not clearly mentioned whether trigone was injected or not. In most of the studies BTX A injections were done under general anaesthesia although some were done under sedation or under local anaesthetic. Duration of follow up varied between 3 and 24 months across studies. All except two studies had patients who were representative of this condition in the population. Out of the 3 RCTs, 2 were of high quality on Jadad scale. The ascertainment of diagnosis, assessment of outcome and the instruments used to assess outcomes were satisfactorily described in all studies. The length of follow up was described in 6/7 studies. Eight studies reported improvement in symptoms. Urodynamic parameters were variable. Meta-analysis was not performed due to heterogeneity in reporting of outcomes. Some adverse events e.g. dysuria and voiding difficulty were noted (19/260 required to self-catheterize at anytime postoperatively). Only one study reported that $7 / 29$ patients needed repeat injections because of recurrence of pain at 6 months

Interpretation of results

Botox injection into the bladder seems to be beneficial for improvement in symptoms in the short term but can be associated with voiding difficulties. The data are heterogenous in terms of definition of the condition, method of injection, definition of cure/improvement and hence this treatment should ideally be used in this condition in research setting.

\section{Concluding message}

There is trend towards short term benefit with intravesical BTX-A injections but further robust evidence should be awaited.

References

1. 7. Giannantoni, A., Porena, M., Costantini, E., Zucchi, A., Mearini, L., \& Mearini, E. (2008) Botulinum A toxin intravesical injection in patients with painful bladder syndrome: 1-year followup. J Urol. 179: 1031-1034.

2. 20. Smith, C. P., Radziszewski, P., Borkowski, A., Somogyi, G. T., Boone, T. B., \& Chancellor, M. B. (2004) Botulinum toxin A has antinociceptive effects in treating interstitial cystitis. Urology 64: 871-875.

\begin{tabular}{ll}
\hline Specify source of funding or grant & None \\
\hline Is this a clinical trial? & No \\
\hline What were the subjects in the study? & HUMAN \\
\hline Was this study approved by an ethics committee? & No \\
\hline This study did not require ethics committee approval because & It is a systematic review and not a primary study \\
\hline Was the Declaration of Helsinki followed? & Yes \\
\hline Was informed consent obtained from the patients? & No \\
\hline
\end{tabular}

